$6 \mathrm{~A}$ M. the house surgeon was called and he found the patient dying. She died twenty-five minutes later. The temperature had been $98^{\circ} \mathrm{F}$. after the operation; at 5 A.M. it had risen to $99 \cdot 2^{\circ}$.

Neeropsy.-The peritoneum was congested and a little fluid was found round the abscess ; the abscess cavity was empty no perforation was discoverea. A large caseous mass occupied the site of the eleventh dorsal vertebra and an abscess containing an ounce of pus was found on the left side. There was "no apparent cause of death."

CASE 10 -A girl aged eight years and a half was admitted to the Paddington-green Children's Hospital on Sept 1st, 1891, under the care of Mr. Watson Cheyne, for treatment of a psoas abscess dependent upon lumbar spinal curvature. on Sept. 3rd she was sick twice and had.abdominal pain, probably the results of the administration of calomel and castor oil. On the 4th the abscess was opened, scraped internally, and irrigated with a 1 in 4000 solution of corrosive sublimate. The cavity was wiped out with sponges, not injected with iodoform, and the wound was sewn up without drainage. After the operation sbe was very pale and restless; she was sick constantly in the evening and during the night and complained of great abdominal pain. The temperature sank to $95.8^{\circ} \mathrm{F}$. One drachm of brandy alternately with champagne was ordered, and one-eighth of a grain of morphia was given subcutaneously. The temperature rose to $982^{\circ}$ at 9 A.M. on the 5 th, but soon fell to $96^{\circ}$. She became quieter, had no pain, but the pulse was very weak and rapid. She was not restless during the 5 th, but vomited frequently, and there was copious diarrhoea, with some blood in the motions. On the 6th the diarrhcea still continued. She had not been sick since the previous afternoon. The right side of the abdomen was very tender. She had no pain, but was very pale and thirsty. The temperature was normal. The pulse was very weak and rapid, but better than on the preceding day. The gums were not sore, nor was there salivation. By the 9th she had gradually improved, the diarrhoea had ceased, the pulse was good and the temperature was normal. She recovered completely without further trouble.

(To be continued.)

\section{TWO RARE VARIETIES OF STRANGULATED INGUINAL HERNIA COMPLICATED BY RETAINED TESTICLE.}

BY GEORGE HEATON, M.B. OXON., F.R.C.S. ENG. ASSISTANT SURGEON TO THE GENERAL HOSPITAL, BIRMINGHAM.

DURIxG the past twelve months, in operating on cases of strangulated hernia, I have met with two varieties which, though well recognised and described, are yet comparatively so rare as to make them worthy of record. Both occurred in patients in whom one testicle had failed to descend completely into the scrotum, and both owed their peculiarity to an arrest in, or deviation from, the normal process of formation of the tunica vaginalis. The following are brief clinical histories and descriptions of the cases.

CASE 1.-Retentio iliaca testis; stranqulated intra-parietal hernia; death from unrelieved intestinal obstruction. - A man twenty-seven years of age was admitted into the General Hospital, Birmingham, on Nov. 10th, 1893, suffering from symptoms of strangulated hernia. His history was that early in the morning of the day of his admission whilst at work he was suddenly selzed with violent abdominal pain. He felt very sick and vomited several times, and then found that his rupture had become much larger than usual, and could no longer be reduced. He had a congenital inguinal hernia on the right side, but he had not worn any truss for seventeen years, as his rupture could always be easily reduced. On admission he complained of violent colicky pain across the epigastrium and tenderness in the right groin. There was a herniz in the right half of the scrotum about the size of a small lemon, but rather more cylindrical in shape than usual. The bernia was tender, dull on percussion, and irreducible, but there was a distinct impulse in it. The right testicle could not be felt. The bowels bad not been moved nor had flatus been passed since the previous day. A hot bath was given and taxis aprlied by the resident surgical officer, but without any effect on the hernia. I performed herniotomy the same evening. The sac, which was unusually thick, was opened, and several ounces of blood-stained fluid escaped. This was washed away and a knuckle of deeply congested gut exposed at the neck of the sac. The intestine was returned without any difficulty, but on attempting to draw it out again for purposes of examination it was found impossible to do so. The sac was dissected away from its connexions, ligatured high up at its neck and excised. No trace of testicle was seen. The wound after being irrigated with perchloride of mercury lotion was closed in the usual manner. He passed a restless nigbt, but when seen on the following day appeared to be comfortable and had lost all his abdominal pain and tenderness. During the next few days he complained of some slight abdominal pain and vomited occasionally. His worst symptom during this time was his continuous restlessness, which was scarcely relieved by opiates. On Nov, 15th there was marked tenderness in the lower part of the abdomen, with rigidity of the abdominal muscles. Pargative enemata were given without relief. The same evening he suddenly became much worse. His pulse was 164, being scarcely perceptible at the wrist; his extremities were cold and blue, and his restlessness extreme. Although he was evidently dying from unrelieved obstruction, his condition was such as to preclude any further operative measures, and he died on the following day.

Necropsy. - There was some general peritonitis in the lower part of the peritoneal cavity and the intestinal coils were much distended. Below and slightly external to the

Diagram 1.

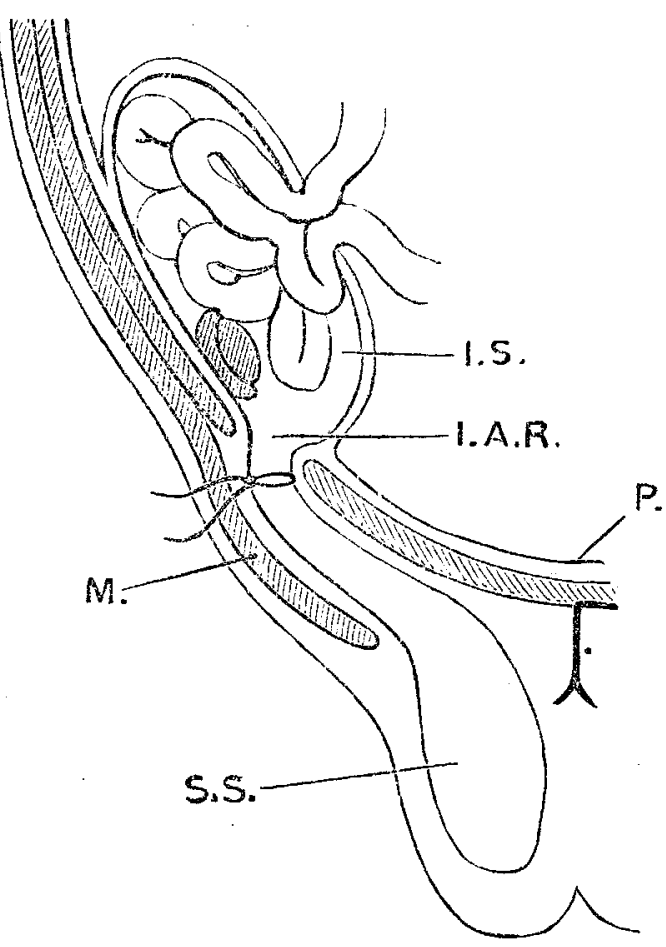

I. S., Intra-parietal sac. I. A. R., Internal abdominal ring. P., Peri toneum. M., Muscle. S. S., Scrotal sac.

internal abdominal ring there was a sac communicating by a small orifice with the abdominal cavity, through which passed a coil of small intestine tightly strangulated. The sac, which was lined throughout by peritoneum, contained some bloody fluid and several coils of intensely black intestine. The testicle, small and atrophied, lay in the outer wall of the sac on the iliac fascia below and external to the internal abdominal ring. The sac communicated by a narrow channel with the stump of the scrotal hernia which had been ligatured, and lay in the subperitoneal tissue on the internal surface of the internal oblique and transversalis muscles.

The position of affairs in this case is best explained by a reference to Diagram 1. The testicle in its course to the scrotum had been, from some cause, arrested in its progress at the internal abdominal ring, and instead of passing along the inguinal canal, had gravitated towards the iliac fossa, dragging with it a pouch of peritoneum, thus forming a large dilated tunica vaginalis lying between the peritoneum and the abdominal parietes ; but an offshoot of this sac passed along the inguinal canal into the scrotum. In this way a sac, lined by peritoneum and containing the testicle, was formed between the peritoneum and the abdominal walls, having a diverticulum which passed into the scrotum. Both sac and diverticulum. 
communicated by a common orifice with the general peritoneal cavity. It was, in other words, one of the varieties of intraparietal hernia, or "hernia en bissac" of French writers. The anatomical peculiarities of the hernia explain several of its clinical peculiarities; they explain the tenseness and irreducibility of the scrotal tumour, and yet the easy reduction of the intestine at the time of operation-the intestine being, of course, only $r \in d u c \in d$ from the diverticulum into the main sac. They also explain the occurrence of a distinct impulse in a hernia tightly strangulated, and the impossibility during operation of withdraning a coil of intestine after reduction for inspection. One can only deplore that the complication present was not suspected or recognised until the patient's condition was such as to preclude any further operative measures for its relief.

CASE 2. Testicle retained in inguinal canal; strangulated encysted hernia; herniotomy; recovery. - A man forty-five years of age was admitted into the General Hospital, Birmingham, on Nov. 20th, 1892, suffering from symptoms of intestinal obstruction of two days' duration. He gave the following history. The left testicle had never been present in the scrotum, but conld formerly be felt in the groin. On several occasions after hard work he had had attacks of acute pain in the retained testicle lasting for several days. For many months past there had been a lax bag of fluid in that groin enveloping the testicle. Two days before admission, whilst at work, be was seized with sudden acute pain in the part ; the pain was much more acute than in previous attacks, and radiated all over the abdomen. Vomiting had been

\section{Diagram 2.}

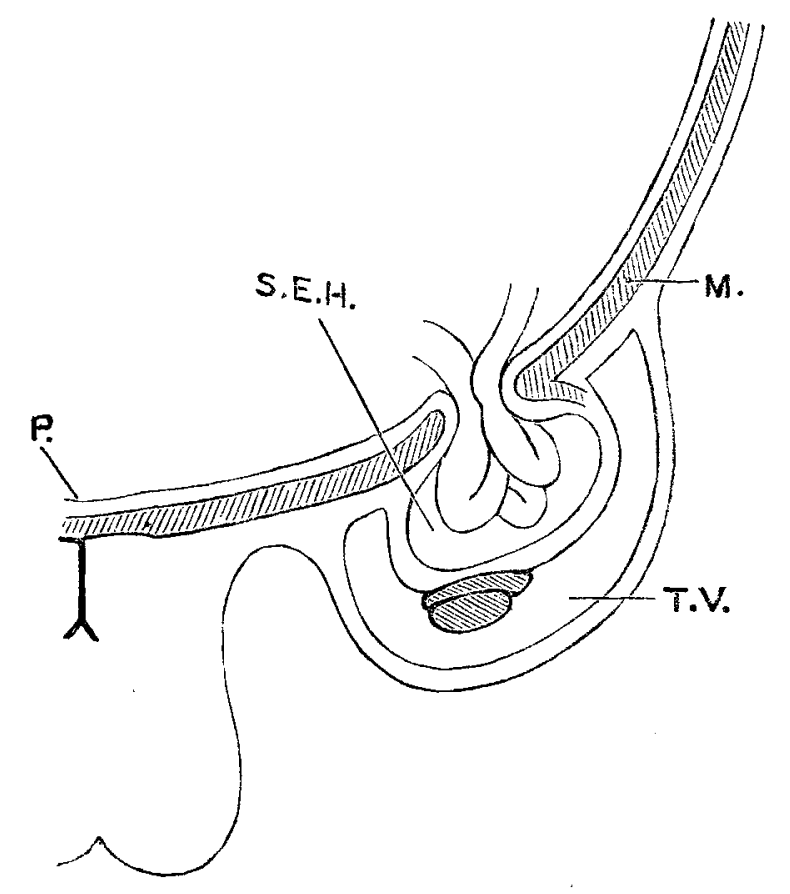

S. E. H., Sac of encysted hernia. P., Peritoneum. M., Muscle. T. V., 1 unica vaginalis.

almost incessant during the last twenty-four hours, and there had been no passage of flatus or fæces in spite of frequent purges. On admiseion he was writhing with acute colicky pains across the epigastrium and vomited frequently. In the left groin was a lax fluid swelling about the size of a large orange. The fluid swelling was neither painf ul nor tender; but through the fluid could be felt a hand, very tender tumour, tense and without impulse. Herniotomy was performed, an incision being made over the most prominent part of the fluid swelling. A sac was opened containing from one to two ounces of clear, straw-coloured hydrocele fluid. A tense, thick-walled tumour was found lying at the bottom of the sac and bulging into it. An atrophied testicle lay attached to its lower and posterior pait. The swelling was incised and opened. About ix drachms of dark-coloured serum escaped and a ragged opening was exposed leading into a third sac, which contained a coil of strangulated small intestine. After reduction of the intestine the whole sac with testicle and cord was dissected away from its connexions up to the internal abdominal ring. The spermatic cord was transfixed and ligatured with silk, and the sac itself, after ligature of its neck, was cat away. The wound was dressed in the usual way. The patient made an uneventfui recovery. The wound was dressed on the following day and the tube removed. It was dressed a second time on the eleventh day, when it was all soundly healed, and the patient left the hospital a few days later well.

This case was evidently a strangulated inguinal hernia, which had in its descent pushed before it the enlarged tunica vaginalis of an undescended testicle as it lay in the ingainal canal; in other words, it was an example of the strangulated encysted hernia of Sir Astley Cooper. A diagrammatic representation of the parts as they Jay in the groin is given in Diagram 2. No difficulty presented itself in diagnosis during the operation. The external sac was so lax that it was felt that it could not be the seat of the symptoms. On opening the second sac the dark blood-stained serum which is met with in tightly strangulated hernix at once escaped, and the intes tine lay exposed at the bottom in a state of strangulation.

Temple row, Birmingham.

\section{THE TREATMENT OF EMPYEMA.}

BY G. A. SUTHERLAND, M.D. EDiN., M.R.C.P. LOND. PHYSICIAN TO THE NORTH LONDON CONSUMPTION HOSPITAL.

Is dealing with the subject of effusions into the pleural cavity it must be remembered that there are certain pecaliari. ties present which distinguish them from ordinary fluid effusions, such as those in the peritoneum or in the kneejoint. Amongst these peculiarities the most important is the elastic retraction of the lungs. In a previous paper I described the importance of this factor in the diagnosis of pleural effusion, pointing out that "there are two distinct stages in pleural effusion, the first while the pressure of the fluid is negative and is held in suspense by the elasticity of the lungs, and the second when the pressure of the fluid is positive, the lung elasticity having been. exhansted. In the first stage there may be varions displacements owing to the elastic tension of the lungs drawing the organs out of their normal position, and in the second stage displacements are due to the pressure of the fluid pushing the organs out of their normal position."

In this paper I shall discuss the importance of the lung elasticity in connexion with the operative treatment of empyema. It is held and asserted by many that as soon as a pleural cavity containing fluid is opened there is a rush of fluid through the aperture owing to the recoil of the parietes, which hase been distended by the effusion. Dr. Goodhart, writing on empyema, says: "In recent cases, directly the pus is let out the tension is relieved, and there is a rush towards the opening; the ribs fall in a little, the diaphragm goes up, themediastinum yields, the other lung somewhat also, and all the parts press shoulder to shoulder in the same way as the surrounding parts drop down, as the bladder contracts when the urine is voided." 2 There are others who hold that this rush of fluid through the opening occurs only in exceptional cases-namely, when the fluid is very excessive in amount and there is a positive intra-pleural pressure. Such cases are comparatively rare in the present day, because it is now fully recognised that the condition is one of great danger to the patient, and operative treatment has therefore beet: employed before its development. It was not so, however, at the time when relief by puncture was first employed, and' Trousseau, amongst others, had many cases in which the pleural effusion was enormous. In his clinical lectares be thus described the behaviour of the fluid on tapping: "The trocar has penetrated the pleural cavity - a fact ascertained by feeling that its point can be mored about freely in a hollow space. On the withdrawal of the stylet the fluid at first flows slowly, then in a continurous jet, and at last in jerking gushes." 3 He further adds that in many cases there will be no flow at all as long as the patient breatbes quietly. These experiences are distinctly opposed to each other. In the quotation given from Dr. Goodhart's writings the tluid is described as being in a state of tension, and its

1 The Physics and Diagnosia of Pleumal Effusion. THe Larcst July 22nd, 1893.

2 On the Behaviour of Fluid in the Chest and the Treatment of Empyema. Brit Mtd. Jour., vol. i. 1887, p. $12 n 3$.

Trousseru : Clinical Medicine, vul. iii., p. 273. Cf. also Fraentzel" p. 696. 